\title{
ACTION DE LA MATIÈRE ORGANIQUE ET DES ENGRAIS MINÉRAUX EN PÉPINIÈRE SUR LA CROISSANCE ET LES CARACTÈRES FOLIAIRES DU DOUGLAS
}

\author{
Ph. LEROY, G. LÉVY et D. G. STRULLU \\ avec la collaboration technique de \\ G. Courrier et M. Guillemette \\ Centre régional de la Propriété forestière, Lorraine-Alsace, 57000 Metz - Le Ban St-Martin \\ Station de Recherches sur les Sols forestiers et la Fertilisation, \\ Centre national de Recherches forestières, I.N.R.A., \\ Champenoux 54370 Einville \\ Laboratoire de Botanique L. Daniel, \\ Faculté des Sciences biologiques \\ 35031 Rennes cedex
}

\section{RÉSUMÉ}

Une expérience, menée sur repiqués de Douglas, combine des apports minéraux et organiques.

La présence d'humus dans le sol de départ est nécessaire, et elle suffit (apports supplémentaires inutiles), au moins durant deux cycles de deux ans, pour assurer une croissance maximale en pépinière; par contre un apport supplémentaire de matière organique en pépinière facilite le démarrage après plantation sur le terrain.

En ce qui concerne la fertilisation minérale, l'optimum se situe entre un taux égal aux exportations et ce même taux duquel on soustrait la totalité des éléments apportés par la matière organique.

En outre, cette expérience montre que les différences de croissance en hauteur en pépinière sont en accord avec la variabilité, suivant les traitements, des caractères morphologiques, anatomiques et chimiques des feuilles.

\section{I. - INTRODUCTION}

Un sol de pépinière s'appauvrit régulièrement en éléments nutritifs par l'exportation de matière végétale et en matière organique par minéralisation de l'humus. Il paraît donc logique d'effectuer des apports divers destinés à compenser cet appauvrissement. S'il est évident qu'il convient de reconstituer la réserve du sol en éléments nutritifs, il n'est pas sûr 
que la présence de matière organique soit toujours utile; il est même probable que dans certaines conditions, substratum formé d'éléments grossiers et apports d'engrais minéraux sous certaines formes lentement solubles, elle soit inutile. Il convient donc surtout d'expliciter le rôle de la matière organique. A ce titre notre expérimentation a pour objet essentiel de répondre aux questions suivantes :

1) La présence de matière organique est-elle utile à un sol de pépinière? Quel est le rôle de cette matière organique indépendamment de l'apport d'éléments nutritifs libérés par sa minéralisation? Ce rôle éventuel peut s'expliquer par l'amélioration de la structure, par l'action sur la mycorhization, la stimulation de la vie microbienne, la libération progressive d'azote minéral, l'adsorption des éléments assimilables.

2) Dans la mesure où la matière organique s'avère utile, quelle est la quantité initiale à apporter? Comment maintenir la quantité optimale? Ceci dépend de plusieurs variables : taux de minéralisation de l'humus du sol, coefficient iso-humique de la matière organique apportée, etc...

3) Les éléments nutritifs libérés par la minéralisation sont-ils tout ou en partie utilisés pour la nutrition des plants? Pratiquement, le fait d'effectuer des apports périodiques de matière organique permet-il de réduire, et dans quelle mesure, les apports d'engrais minéraux?

4) Le fait d'effectuer des apports trop importants d'éléments nutritifs (exportations compensées entièrement par des apports minéraux sans tenir compte des apports organiques) ne risque-t-il pas, à long terme, de provoquer une élévation du taux de saturation et du $\mathrm{pH}$ du sol dangereuse pour des résineux?

L'ensemble des renseignements concernant les points qui viennent d'être évoqués ne pourra être fourni qu'à long terme en raison surtout de la lenteur de la minéralisation de l'humus du sol. Cette remarque, valable en partie pour les points 1.2 et 4 , l'est encore plus pour le point 3 ; en effet, pendant les premières années de l'expérience, l'humus minéralisé pendant chaque cycle provient surtout de l'humus de départ du sol et non de la matière organique apportée : il est donc impossible de connaître exactement les quantités d'éléments nutritifs libérés par minéralisation de l'humus.

Le problème de l'évaluation de l'influence des différents traitements sur le comportement des plants nous a amené à effectuer certaines études dont les résultats constituent un second pôle d'intérêt de cet article. En effet, le but recherché dans une pépinière est de produire des plants grands et vigoureux, et « démarrant » bien après plantation. La croissance en hauteur des plants est un critère souvent utilisé, mais est-il bien représentatif de l'état général et des potentialités des plants? Donc, en plus d'une étude morphologique assez poussée, ainsi que d'analyses foliaires, nous avons étudié en détail l'anatomie foliaire. Cela nous a permis non seulement de comparer l'effet des divers traitements sur chaque caractère étudié, résultats intéressants en soi, mais également de tenter une synthèse pour l'ensemble du comportement des plants, y compris la croissance après plantation.

Au départ de l'expérimentation en pépinière des plantules de 1 an ont été repiquées dans les bâches où étaient répartis les divers traitements. Chaque « cycle » de l'expérience se poursuit pendant 2 ans en pépinière. Les plants 1-2 ont été plantés ensuite sur le terrain. Nous présentons ici les résultats obtenus pour les 2 premiers cycles en pépinière (1967-68 et 1969-70) et les premiers résultats acquis en forêt. 


\section{MATÉRIEL ET MÉTHODES}

L'expérience fut conduite en pépinière à Amance (54) dans 5 bâches de $30 \mathrm{~m}^{2}$ chacune.

Ces bâches ont été remplies, sur une hauteur de $40 \mathrm{~cm}$, soit d'un matériau formé surtout de sables fins $(76 \%)$ issu de carrière sur rhétien (forêt de Bezanges 54$)$ et pratiquement sans matière organique $(0,3 \%)$, soit d'un horizon A1 (mull acide à $5 \%$ de matière organique en moyenne) de sol brun acide sur même substrat et de même composition granulométrique. En fait la teneur en matière organique est assez variable d'un endroit à l'autre.

Ces substrats sont pauvres chimiquement. Leur structure, en l'absence d'humus, est battante; elle devrait donc être améliorée par la matière organique. La matière organique choisie pour effectuer les apports périodiques est une terre de bruyère provenant de Sologne; il était nécessaire, en effet, pour une pépinière de résineux de prendre une matière organique acide. Cette terre de bruyère contient environ $50 \%$ de matière organique, son $\mathrm{pH}$ est de 3,4 ; l'attaque sulfurique fournit les valeurs suivantes (éléments « totaux ») : $1,3 \%$ de N, $0,36 \%$ de $\mathrm{P}_{2} \mathrm{O}_{5}, 0,23 \%$ de $\mathrm{K}, 0,26 \%$ de $\mathrm{Ca}$, $0,03 \%$ de $\mathrm{Mg}$. Il s'agit de matière organique déjà en bonne partie humifiée; son coefficient isohumique est probablement peu inférieur à 1 .

Les engrais utilisés ont été les suivants : superphosphate ordinaire; sulfate de $\mathrm{K}$ (1 ${ }^{\mathrm{er}}$ cycle), patentkali ( $2^{\mathrm{e}}$ cycle), chaux éteinte, sulfate d'ammonium (1 ${ }^{\text {re }}$ année de chaque cycle), ammonitrates ( $2^{\mathrm{e}}$ année).

Les apports d'engrais azotés étaient subdivisés : 1 ou 2 apports pendant la $1^{\text {re }}$ année $(1 / 3$ de la dose totale) et 2 apports la $2^{\mathrm{e}}$ année ( $2 / 3$ de la dose totale).

Chaque modalité combine un traitement organique et un traitement minéral.

Les 4 traitements organiques utilisés sont les suivants :

- $\mathrm{OO}=$ pas de matière organique dans le sol (substrat issu de carrière, cf. plus haut), pas d'apport organique;

- $\mathrm{O}=$ substrat formé de sable fin humifère (cf. plus haut), pas d'apport organique;

- $\mathrm{T} 1=$ même substrat que $\mathrm{O}$, apport de terre de bruyère, au début de chaque cycle, destiné à compenser une minéralisation de $2 \%$ par an de l'humus du substrat (apport de $80 \mathrm{~kg} /$ are de matière organique sèche ou $160 \mathrm{~kg} /$ are de terre de bruyère sèche);

- $\mathrm{T} 2=$ même substrat que $\mathrm{O}$, apport de terre de bruyère double de $\mathrm{T} 1$ destiné à compenser une minéralisation de $4 \%$ par an.

Le taux de minéralisation réel est probablement compris entre 2 et $4 \%$; il n'est sans doute pas inférieur à $2 \%$ car les conditions sont favorables à la minéralisation (arrosage assez fréquent).

Les 4 traitements minéraux utilisés sont les suivants :

- $\mathrm{O}$ : pas d'apport d'engrais minéral;

- F2 : apport d'engrais destiné à compenser exactement les exportations d'éléments minéraux lors de l'arrachage des plants à la fin de chaque cycle et effectué, sauf pour $\mathrm{N}$, avant le repiquage Les chiffres d'exportations réelles, établis à la fin du $1^{\text {er }}$ cycle, se sont avérés très voisins des chiffres retenus au départ;

- F1 : apport d'engrais minéraux calculé de telle sorte que pour chaque élément la quantité apportée par F1 ajoutée à celle apportée par T1 fasse une somme égale à celle apportée par F2 (c'est-à-dire exportée);

- $F^{\prime} 1$ : apport calculé de la même manière que $F 1$, mais en partant des quantités d'éléments amenées par T2;

En réalité, si les doses $\mathrm{F} 1$ et $\mathrm{F}^{\prime} 1$ du $2^{\mathrm{e}}$ cycle correspondent bien à la définition précédente, il n'en est pas de même pour le $1^{\text {er }}$ cycle : par suite d'une erreur (hétérogénéité dans les livraisons de terre de bruyère), elles sont nettement supérieures à ce qu'elles devraient être.

La valeur de ces apports est indiquée au tableau 1 pour les 3 éléments essentiels. On peut remarquer que pour $\mathrm{F} 1$ et $\mathrm{F}^{\prime} 1$ les apports effectués lors du $2^{\mathrm{e}}$ cycle sont nettement inférieurs à ceux du $1^{\text {er }}$ cycle. Les apports de chaux éteinte ont été calculés en tenant compte du calcium amené par le superphosphate.

L'expérience comporte les 8 modalités suivantes (le traitement organique esí indiqué en premier, l'apport d'engrais minéral ensuite) :

$\begin{array}{lll}x & x & \text { OOF2 } \\ x & x & \text { OF2 } \\ \text { T10 } & \text { T1F1 } & \text { T1F2 } \\ \text { T20 } & \text { T2F } 1 & \text { T2F2 }\end{array}$


TABLEAU 1

Apports minéraux pour les divers traitements

Mineral supplies for the various treatments

\begin{tabular}{c|c|c|c}
\hline & $\begin{array}{c}\mathrm{N} \\
(\mathrm{kg} / \mathrm{are})\end{array}$ & $\begin{array}{c}\mathrm{P}_{2} \mathrm{O}_{5} \\
(\mathrm{~kg} / \mathrm{are})\end{array}$ & $\begin{array}{c}\mathrm{K}_{2} \mathrm{O} \\
(\mathrm{kg} / \mathrm{are})\end{array}$ \\
\hline $\mathrm{F} 2$ & 2,3 & 0,70 & 1,10 \\
\hline $\begin{array}{c}\mathrm{F} 1 \\
\left(1^{\mathrm{er}} \text { cycle }\right)\end{array}$ & 1,6 & 0,42 & 0,74 \\
\hline $\begin{array}{c}\mathrm{F}^{\prime} 1 \\
\left(1^{\mathrm{er}} \text { cycle }\right)\end{array}$ & 1,2 & 0,25 & 0,38 \\
\hline $\begin{array}{c}\mathrm{F} 1 \\
\left(2^{\mathrm{e}} \text { cycle }\right)\end{array}$ & 0,3 & 0,12 & 0,65 \\
\hline $\begin{array}{c}\mathrm{F}^{\prime} 1 \\
\left(2^{\mathrm{e}} \text { cycle }\right)\end{array}$ & 0 & 0 & 0,20 \\
\hline \hline
\end{tabular}

Il s'agit d'une expérience factorielle incomplète; en effet toutes les combinaisons ne sont pas représentées, parce que certaines n'offraient a priori guère d'intérêt et aussi à cause du manque de place. C'est une expérience en blocs, chacune des 5 bâches représentant un bloc.

A la fin de chaque cycle, on a effectué une plantation sur le terrain, en forêt domaniale de Bezanges sur le sol brun acide d'où provenait le substratum utilisé en pépinière. Seules trois modalités ont pu être plantées à l'issu du $1^{\text {er }}$ cycle, choisies d'après les résultats de pépinière, alors que toutes ont pu l'être à la fin du $2^{\mathrm{e}}$ cycle. Les plantations furent effectuées en blocs. Les mesures ont été effectuées à l'automne 1972 .

Avant d'énoncer les résultats obtenus il convient de dire que ces résultats ne sont à priori valables que dans les conditions précises de la présente expérimentation.

$$
\text { III. - RÉSULTATS }
$$

\section{1. - Résultats du ler $^{\mathrm{er}}$ cycle en pépinière}

\subsection{1. - La croissance en hauteur.}

\section{Hauteur fin 1968 (fin 2e année).}

Le test n'est pas significatif ( $\mathrm{F}$ calculé $=1,84, \mathrm{~F}$ théorique à $5 \%=2,36$ ). La hauteur des divers traitements en $\mathrm{cm}$, est la suivante :

$$
\begin{aligned}
& \mathrm{T} 1 \mathrm{~T} 1>\mathrm{T} 1 \mathrm{~F} 2>\mathrm{T} 2 \mathrm{~F}^{\prime} 1>\mathrm{T} 2 \mathrm{~F} 2>\mathrm{OF} 2>\mathrm{T} 1 \mathrm{O}>\mathrm{OOF} 2>\mathrm{T} 2 \mathrm{O} \\
& \begin{array}{lllllll}
60,10 & 59,20 & 56,94 & 53,91 & 52,98 & 50,08 & 48,34
\end{array}
\end{aligned}
$$

Remarquons seulement que les traitements recevant à la fois des apports organiques et minéraux se classent tous en tête tandis que ceux qui ne comportent qu'un seul type de fertilisant se trouvent en queue.

L'analyse de covariance par contre fait apparaître les différences significatives suivantes :

- pour les traitements organiques à $5 \%: \mathrm{OO}<\mathrm{T} 1$ 
- pour les traitements minéraux

$$
\begin{aligned}
& \text { à } 1 \%: \mathrm{O}<\mathrm{F} 1 \text { et } \mathrm{F}^{\prime} 1 \\
& \text { à } 5 \%: \mathrm{O}<\mathrm{F} 2
\end{aligned}
$$

L'apport F2 n'est pas préférable aux apports $F 1$ et $\mathrm{F}^{\prime} 1$.

L'absence de tout apport minéral et l'absence de matière organique dans le sol se sont donc avérés néfastes pour l'ensemble de la croissance des plants au cours des deux années passées en pépinière.

Pousse 1967 (1 $1^{\text {re }}$ année).

Le classement des traitements est très différent de ce qu'il est fin 1968. Les apports de matière organique se sont avérés (significativement) défavorables, en particulier T2.

Cependant, les plants du traitement OOF2, bien que les plus grands, présentaient un aspect jaunâtre et grêle.

Pousse 1968 (2 $2^{\mathrm{e}}$ année).

Les résultats sont presque équivalents à ceux concernant la hauteur fin 1968; seule différence à signaler : $\mathrm{OO}<\mathrm{T} 2$, significativement. Le traitement $\mathrm{OOF} 2$ a nettement la pousse la plus faible de toutes.

Le classement des traitements pour la hauteur fin 1968 est donc nettement plus influencé par la pousse 1968 que par la pousse 1967, ceci est normal. L'effet des traitements sur la croissance en hauteur a été très différent au cours des 2 années, principalement en ce qui concerne l'action de la matière organique.

\subsection{2. - Autres caractères de croissance.}

Ces mesures ont été effectuées sur un échantillonnage basé sur la taille des plants en 1968.

\section{Diamètre au collet.}

Cette mesure correspondant au diamètre d'un organe âgé de 3 ans ne donne pas de résultats significatifs; les moyennes $(\mathrm{en} \mathrm{cm}$ ) suivantes sont très homogènes.

$$
\begin{array}{llllllll}
\text { T2F'1 } & \text { OF2 } & \text { T1F2 } & \text { T1F1 } & \text { T10 } & \text { T20 } & \text { OOF2 } & \text { T2F2 } \\
1,04 & 1,01 & 1,01 & 0,98 & 0,96 & 0,95 & 0,94 & 0,93
\end{array}
$$

Diamètre de la pousse de l'année du prélèvement.

Le test $\mathrm{F}$ est significatif à $5 \%(\mathrm{~F}=2,28)$. Un test de Duncan sur ce caractère montre que OOF2 $=0,38 \mathrm{~cm}$ se détache largement des autres traitements qui se classent ainsi :

$$
\begin{array}{llllll}
\text { OF2 } & \text { T10 } & \text { T1F2 } & \text { T2F2 } & \text { T1F1 } & \text { T2F'1 } \\
0,49 & 0,50 & 0,50 & 0,51 & 0,53 & 0,55
\end{array}
$$

\section{Caractère des bourgeons.}

Tous les traitements offrent des résultats équivalents en ce qui concerne le nombre de bourgeons sur la flèche et le diamètre du bourgeon terminal. 


$\begin{array}{lccccccccc}\text { Nombre : OOF2 } & \text { T20 } & \text { T10 } & \text { OF2 } & \text { T2F2 } & \text { T1F1 } & \mathrm{T}_{2} \mathrm{~F}^{\prime} 1 & \mathrm{~T} 1 \mathrm{~F} 2 \\ 8 & 9 & 9 & 9 & 10 & 10 & 10 & 11 \\ \text { Diamètre : T10 } & \text { OOF2 } & \mathrm{T} 20 & \mathrm{~T} 1 \mathrm{~F} 1 & \mathrm{~T} 2 \mathrm{~F} 2 & \mathrm{~T} 2 \mathrm{~F}^{\prime} 1 & \mathrm{~T} 1 \mathrm{~F} 2 & \mathrm{OF} 2 \\ \text { en cm : } & 0,48 & 0,50 & 0,50 & 0,51 & 0,51 & 0,52 & 0,53 & 0,56\end{array}$

\subsection{3. - Caractères foliaires.}

a) Étude des caractères pris isolément.

Les résultats des tests $\mathrm{F}$ significatifs sont présentés dans la figure $1^{1}$. Ces valeurs de $\mathrm{F}$ sont portées en face de chaque caractère. Les moyennes obtenues pour chaque caractère significatif ou non, sont portées en annexe I.

Dans la figure 1 les traitements (triangles) sont rangés selon l'axe I montrant le rôle de la matière organique ( $\mathrm{F} 2$ étant constant) et selon les axes II et III montrant le rôle des engrais minéraux ( $\mathrm{T} 1$ ou $\mathrm{T} 2$ étant constants).

Dans un axe un caractère donné (case) d'un traitement donné (triangle) est porté en noir s'il offre une différence significative positive (par test $F$ et test de Duncan), avec son homologue du traitement précédant dans l'axe considéré.

La lecture de cette planche apporte les données suivantes :

- Axe I : Action de la matière organique.

Le traitement OOF2 est le moins favorable au développement des jeunes Douglas. Tous les caractères foliaires peuvent être améliorés.

Le traitement OF2 diffère du précédent (OOF2) par la présence d'humus. Dans ce cas l'on enregistre la progression de la taille des feuilles de première année et de deuxième année, de la largeur de l'épiderme inférieur de première année, du nombre de rangées de stomates et du nombre de cellules de l'endoderme de deuxième année.

Le traitement $\mathrm{T} 1 \mathrm{~F} 2$ correspondant à $\mathrm{OF} 2$ fertilisé par $160 \mathrm{~kg}$ de terre de bruyère sèche produit d'une part un nouvel accroissement significatif de la taille des feuilles de première année et d'autre part, une progression des caractères non modifiés par OF2.

Le traitement $\mathrm{T} 2 \mathrm{~F} 2$ qui apporte $320 \mathrm{~kg}$ de terre de bruyère ne provoque aucune amélioration des caractères, au contraire beaucoup d'entre eux ne sont pas différents de OOF2.

- Axe II : Action de la fertilisation minérale, T1 constant.

Le traitement T10 diffère peu de OOF2 alors que le traitement T1F1 induit une augmentation de la valeur de tous les caractères foliaires. T1F2 est équivalent à T1F1.

- Axe III : action de la fertilisation minérale T2 constant

Les traitements $\mathrm{T} 20, \mathrm{~T} 2 \mathrm{~F}^{\prime} 1, \mathrm{~T} 2 \mathrm{~F} 2$ sont peu différents les uns des autres ainsi que de T10 et de OOF2.

1. Le principe de cette planche a été utilisé par l'un de nous dans un travail antérieur (D. G. STrullu. Thèse de $3^{\mathrm{e}}$ Cycle 1970) dont ce paragraphe reprend certains résultats. 
AXE I Action de $T$

AXE II Action de $F$ avec T1

AXE III Action de $F$ avec T2

Effet non significatif

Effet significatif par rapport à OOF2

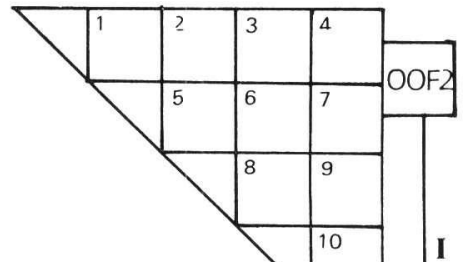

Effet significatif par rapport au traitement précédent dans l'axe considéré

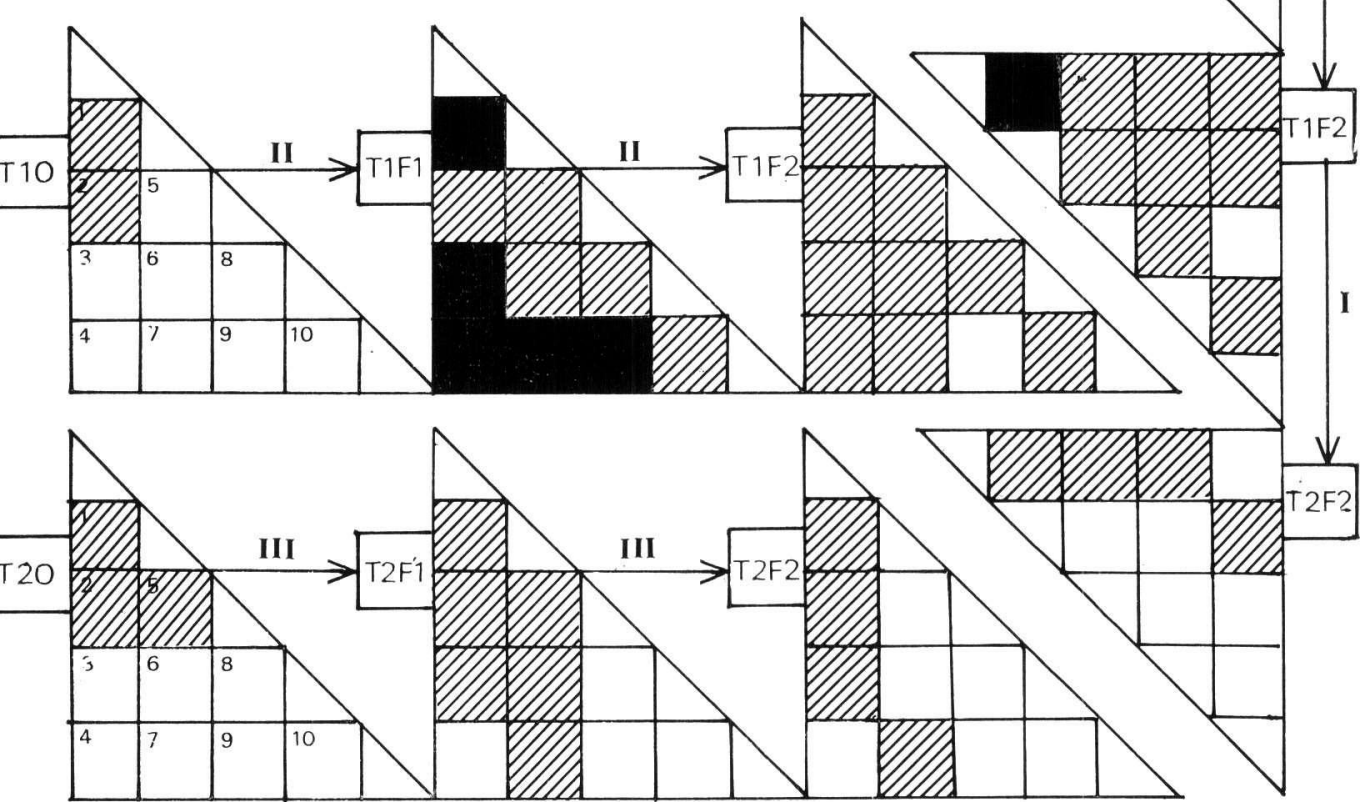

FIG. 1. - Variabilité des caractères foliaires

Variability of foliar features

1 taille des feuilles de $1^{\text {re }}$ année

2 taille des feuilles de $2^{\mathrm{e}}$ année

3 épaisseur des feuilles de $1^{\text {re }}$ année

4 largeur des feuilles de $1^{\text {re }}$ année

5 largeur de l'épiderme inférieur des feuilles de $1^{\text {re }}$ année

6 largeur des bandes stomatiques des feuilles de $1^{\text {re }}$ année

7 hauteur du cylindre central des feuilles de $1^{\text {re }}$ année

8 nombre de rangées de stomates des feuilles de $2^{\mathrm{e}}$ année

9 nombre de cellules de l'hypoderme de $2^{\mathrm{e}}$ année

10 nombre de cellules de l'endoderme des feuilles de $2^{\text {e }}$ année

$\mathrm{x}=$ significatif à $5 \%$

$\mathbf{x x}=$ significatif à $1 \%$

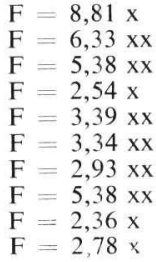


A la suite de cette analyse on peut énoncer que dans nos conditions expérimentales : feuilles;

- chaque type de fertilisation (minérale et organique) influence le développement des

- la présence d'humus, même sans apport organique a un effet très significatif;

- une fertilisation minérale est très bénéfique surtout si l'apport de matière organique n'est pas trop élevé.

b) Étude des caractères pris dans leur ensemble

Les résultats sont traités en deux groupes : les feuilles de $1^{\text {re }}$ année et les feuilles de $2^{\mathrm{e}}$ année en pépinière.

Une analyse canonique effectuée sur ces caractères donne des résultats significatifs.

Les caractères morphologiques des feuilles (longueur, largeur, épaisseur), sont liés (positivement) entre eux ainsi qu'à la largeur des bandes stomatiques (dont dépend le nombre de rangées de stomates et le nombre de stomates). Par contre, largeur de la nervure et nombre de cellules de l'hypoderme paraissent assez fixes, peu liés à la dimension des feuilles.

Compte tenu de ces corrélations entre les différents caractères, tant la première que la deuxième année, l'analyse canonique indique que les variations se font selon une seule direction significative $y_{1}$.

Les classements sont déterminés essentiellement par la taille des feuilles.

La valeur relative des 2 premières composantes principales pour les différents traitements est représentée sur la figure 2 , pour les feuilles de $2^{\mathrm{e}}$ année. Le graphique est peu différent pour celles de $1^{\text {re }}$ année.

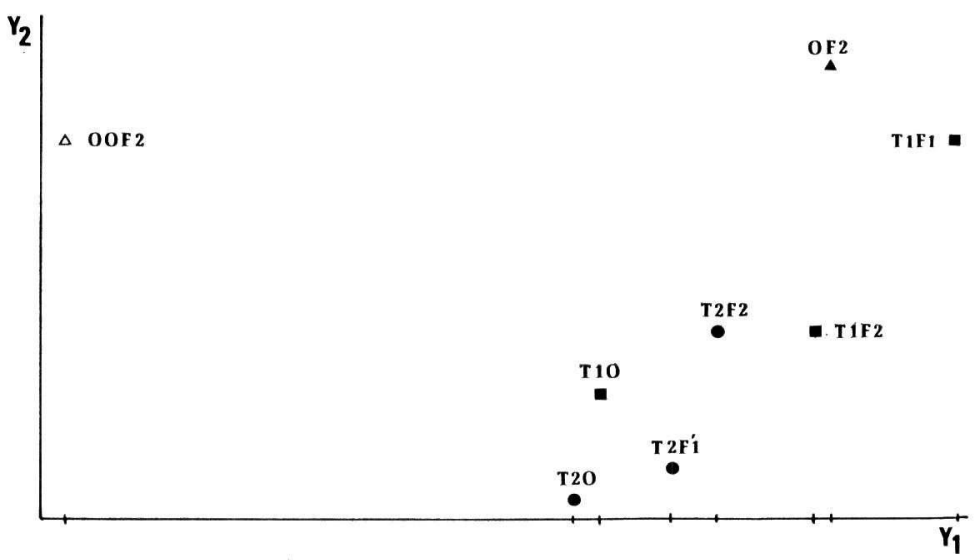

FIG. 2. - Graphique des composantes principales pour les caractères des feuilles de $2^{\mathbf{e}}$ année Graphic of main components for the features of second year leaves 
Ces graphiques appellent les commentaires suivants :

- La première et la deuxième année, OOF2 se situe dans les valeurs minimales. Ce traitement est nettement séparé de OF2. Ainsi la seule présence d'humus a une action importante sur les caractères foliaires.

- le traitement T10 se situe toujours dans les valeurs plutôt moyennes des composantes $\left(\mathrm{Y}_{1}\right)$. T1F1 est dans les valeurs maximales. Donc au taux $\mathrm{T} 1=160 \mathrm{~kg} /$ are de terre de bruyère, la fertilisation minérale joue un rôle important. Par contre, T1F1 et T1F2 sont assez voisins des valeurs maximales. Un apport d'engrais supplémentaire (différence F2 - F1 cf. protocole) n'a pas eu d'action.

- les traitements $\mathrm{T} 10$ et T20 sont dans des valeurs plutôt moyennes des composantes principales $\left(\mathrm{Y}_{1}\right)$. Une fertilisation $\mathrm{T} 2 \mathrm{n}$ 'a pas d'effet différent de $\mathrm{T} 1$ et une fertilisation organique abondante ne peut donc pas suppléer une fertilisation minérale insuffisante.

- les traitements $\mathrm{T}_{2} \mathrm{~F}^{\prime} 1$ et $\mathrm{T} 2 \mathrm{~F} 2$ sont inférieurs à $\mathrm{T} 1 \mathrm{~F} 1$ et $\mathrm{T} 1 \mathrm{~F} 2$; ainsi tous les traitements en T2 sont groupés alors que les traitements en T1 sont plus dispersés. Donc, par rapport à un taux $\mathrm{T} 1$, un taux $\mathrm{T} 2$ de matière organique limite les effets de la fertilisation minérale F1 ou F2 sur les caractères foliaires.

\subsection{4. - Enracinement.}

Lors du repiquage des plants la racine fut habillée; dans aucun cas elle ne s'est allongée. Dans tous les cas de nombreuses racines secondaires sont apparues.

Sur la base des résultats d'une évaluation des racines par une échelle de 1 à 4 nous pouvons dire que T2 $F^{\prime} 1$ présente le moins de racines, que T10 et T20 offrent le meilleur enracinement et enfin que OOF2, OF2, T1F2, T2F2 sont équivalents.

En outre la même méthode appliquée aux racines courtes mycorhyzées indique la supériorité de T10 et T20 sur les autres traitements.

Il convient de préciser que dans tous les traitements les propriétés physiques du sol sont voisines. Ces propriétés physiques sont assez favorables au développement des racines; les différences entre traitements sont réduites.

En résumé, dans nos conditions de culture tous les plants possèdent un système racinaire bien développé. Les traitements fertilisés par la terre de bruyère présentent cependant un chevelu racinaire plus dense. L'observation de ce chevelu racinaire nous a permis de mettre en évidence à côté des racines longues exploratrices du sol des racines courtes mycorrhizées.

\subsection{5. - Nutrition.}

- Fin $1^{\text {re }}$ année : analyse non effectuée;

- Fin $2^{\mathrm{e}}$ année : (tableau 2).

On remarque principalement des teneurs en $\mathrm{N}$ nettement inférieures à l'optimum surtout pour OOF2, mais également pour T10 et T20. 
TABLEAU 2

Analyses foliaires fin $1^{\mathrm{er}}$ cycle

Foliar analysis at the end of first cycle

\begin{tabular}{c|c|c|c|c}
\hline \hline & $\mathrm{N} \%$ & $\mathrm{P} \%$ & $\mathrm{~K} \%$ & $\mathrm{Ca} \%$ \\
\hline OOF2 & 1,23 & 0,17 & 0,61 & 0,40 \\
\hline OF2 & 2,07 & 0,16 & 0,58 & 0,49 \\
\hline $\mathrm{T} 10$ & 1,36 & 0,23 & 0,61 & 0,40 \\
\hline $\mathrm{T} 1 \mathrm{~F} 1$ & 2,12 & 0,20 & 0,61 & 0,53 \\
\hline $\mathrm{T} 1 \mathrm{~F} 2$ & 2,21 & 0,25 & 0,64 & 0,50 \\
\hline $\mathrm{T} 20$ & 1,39 & 0,22 & 0,68 & 0,45 \\
\hline $\mathrm{T} 2 \mathrm{~F}^{\prime} 1$ & 1,92 & 0,23 & 0,67 & 0,49 \\
\hline $\mathrm{T} 2 \mathrm{~F} 2$ & 1,95 & 0,21 & 0,65 & 0,50 \\
\hline \hline
\end{tabular}

3.16. - Remarques sur les résultats obtenus pendant le l $^{\mathrm{er}}$ cycle.

La remarque la plus évidente concerne OOF2 : ce traitement est nettement le plus défavorable de tous pour la croissance en longueur et en diamètre, les caractères foliaires et la nutrition. La pousse 1967 fait très nettement exception, mais l'aspect jaune des plants (des feuilles) et les caractères foliaires sont en contradiction avec la longueur de leur pousse. Tous les résultats obtenus en 1968 pour l'appareil aérien concordent; les traitements offrant la nutrition la plus défavorable provoquent la plus faible croissance en hauteur et les caractéristiques morphologiques foliaires les moins développés. Pour l'appareil racinaire cette concordance n'apparaît pas toujours; le problème mérite d'être approfondi.

\section{2. - Résultats du $2^{\mathrm{e}}$ cycle en pépinière}

Rappelons que les apports $\mathrm{F} 1$ et $\mathrm{F}^{\prime} 1$, correspondant aux quantités d'éléments nutritifs retenus pour le deuxième cycle sont plus faibles que ceux du premier cycle.

\subsection{1. - Croissance en hauteur.}

Hauteur fin 1970 (fin $2^{\mathrm{e}}$ année) (tableau 3).

Le test $F$ est très significatif. Les trois meilleurs traitements, qui sont ceux recevant la dose $F_{2}$ (sauf OOF2), sont très significativement différents des cinq autres. OOF2 est intermédiaire entre les trois meilleurs et les quatre plus mauvais traitements. 
TABLEAU 3

Hauteur fin $1970(\mathrm{~cm})$

Height of the plants at the end of 1970 (in $\mathrm{cm}$ )

\begin{tabular}{|c|c|c|c|c|c|c|c|c|}
\hline $\begin{array}{c}\mathrm{T}_{2} \mathrm{~F}^{\prime} 1 \\
48,3\end{array}$ & $\begin{array}{c}\text { T10 } \\
48,87\end{array}$ & $\begin{array}{c}\text { T20 } \\
49,32\end{array}$ & $\begin{array}{l}\text { T1F1 } \\
52,28\end{array}$ & $\begin{array}{l}\text { OOF2 } \\
54,50\end{array}$ & $\begin{array}{l}\text { T2F2 } \\
64,80\end{array}$ & $\begin{array}{l}\text { T1F2 } \\
64,81\end{array}$ & $\begin{array}{c}\text { OF2 } \\
66,19\end{array}$ & \\
\hline & & & & $x x$ & $x x$ & $\mathrm{xx}$ & $\mathrm{xx}$ & $\mathrm{T} 2 \mathrm{~F}^{\prime} 1$ \\
\hline & & & & $\mathrm{x}$ & $x x$ & $\mathrm{xx}$ & $x x$ & $\mathrm{~T} 10$ \\
\hline & & & & $\mathrm{x}$ & $x x$ & $\mathrm{xx}$ & $\mathrm{xx}$ & T20 \\
\hline & & & & & $x x$ & $\mathrm{xx}$ & $\mathrm{xx}$ & $\mathrm{T} 1 \mathrm{~F} 1$ \\
\hline & & & & & $x x$ & $\mathrm{xX}$ & $\mathrm{xx}$ & OOF2 \\
\hline & & & & & & & & $\mathrm{T} 2 \mathrm{~F} 2$ \\
\hline & & & & & & & & $\mathrm{T} 1 \mathrm{~F} 2$ \\
\hline
\end{tabular}

La discrimination s'est donc effectuée essentiellement d'après le critère : présence ou absence de F2, et en second lieu suivant la présence ou non d'humus au départ dans le sol.

Les différences par rapport à la hauteur des plantes à la fin du $1^{\text {er }}$ cycle (fin 1968) s'expliquent probablement par la différence des apports $\mathrm{F} 1$ et $\mathrm{F}^{\prime} 1$ lors des deux cycles.

Le classement des traitements en fonction de la teinte des aiguilles concorde parfaitement avec celui de la taille des plants.

Pousse 1969 (1 $1^{\text {re }}$ année).

La différence essentielle par rapport à la hauteur fin 1970 concerne OOF2 dont les plants ont la pousse nettement la plus faible de toutes.

Pousse 1970 ( $2^{\mathrm{e}}$ année).

Les résultats sont très voisins de ceux qui concernent la hauteur fin 1970.

\subsection{2. - Autres caractères morphologiques.}

L'étude d'un échantillonnage effectué lors de l'arrachage des plants (1-2) offre les moyennes suivantes pour la longueur des feuilles en $\mathrm{cm}$ :

$\begin{array}{cccccccc}\text { OOF2 } & \text { OF2 } & \text { T10 } & \text { T1F1 } & \text { T1F2 } & \text { T20 } & \text { T2F'1 }^{\prime} & \text { T2F2 } \\ 3,2 & 3,4 & 3,0 & 2,9 & 3,7 & 3,0 & 2,9 & 3,3\end{array}$


Ces résultats montrent d'une part que pour le deuxième cycle le caractère discriminant est la présence ou l'absence de $F 2$, les taux $F 1$ et $F^{\prime} 1$ (différents de $F 1$ et $F^{\prime} 1$ du premier cycle, voir protocole) n'assurant pas un développement foliaire maximum, et d'autre part que les variations de la longueur des feuilles en fonction des traitements concordent bien avec les variations de la croissance en hauteur, résultat déjà apparu lors du premier cycle.

En outre, les études du système racinaire, limitées pour des raisons techniques, n'ont pas montré de variations significatives entre traitements.

\subsection{3. - Nutrition.}

Fin 1969 (1 ${ }^{\text {re }}$ année).

Elle semble excellente pour tous les traitements.

Fin 1970 (2e année) (tableau 4).

TABLEAU 4

Analyses foliaires fin $2^{\mathrm{e}}$ cycle

Foliar analysis at the end of second cycle

\begin{tabular}{c|c|c|c|c}
\hline \hline & $\mathrm{N} \%$ & $\mathrm{P} \%$ & $\mathrm{~K} \%$ & $\mathrm{Ca} \%$ \\
\hline OOF2 & 1,61 & 0,22 & 0,96 & 0,32 \\
\hline OF2 & 2,06 & 0,19 & 0,81 & 0,43 \\
\hline $\mathrm{T} 10$ & 1,67 & 0,32 & 0,97 & 0,36 \\
\hline $\mathrm{T} 1 \mathrm{~F} 1$ & 2,02 & 0,34 & 1,04 & 0,40 \\
\hline T1F2 & 2,03 & 0,23 & 0,92 & 0,44 \\
\hline T20 & 2,00 & 0,35 & 0,97 & 0,38 \\
\hline T2F'1 & 1,93 & 0,38 & 1,06 & 0,40 \\
\hline T2F2 & 2,03 & 0,23 & 0,92 & 0,43 \\
\hline \hline
\end{tabular}

On remarque surtout une teneur en $\mathrm{N}$ inférieure à l'optimum pour OOF2 et T10, et peut-être pour $\mathrm{T} 2 \mathrm{~F}^{\prime} 1$ et $\mathrm{T} 20$. Ce résultat est très voisin de celui enregistré à la fin du $1^{\mathrm{er}}$ cycle, compte tenu de la modification apportée à la dose $\mathrm{F}^{\prime} 1$ entre les 2 cycles : il y aurait certaines difficultés de nutrition azotée dans le sol ne contenant pas de matière organique ainsi que dans ceux n'ayant pas reçu d'azote sous forme minérale. 
Il y a concordance entre les résultats de ce test foliaire et la pousse 1969; il n'en est pas de même pour la pousse 1970 du traitement OOF2 : celle-ci est moyenne, alors que le déficit azoté est très marqué.

\subsection{4. - Teneur en matière organique du sol.}

Pour les traitements autres que OOF2, elle varie à la fin du $2^{\mathrm{e}}$ cycle entre 4,82 et $5,78 \%$. Les différences ne sont pas significatives. De plus, elle varie assez sensiblement suivant les prélèvements pour un même traitement.

\section{3. - Comportement après plantation}

3.31. $-1^{\mathrm{er}}$ cycle.

Pour des raisons matérielles, seuls les plants issus de trois traitements ont pu être testés sur le terrain; nous avons choisi OF2 (apport minéral seul, mais présence de matière organique au départ), T10 (apport organique seul) et T1F2 (apport mixte).

Les mesures effectuées quatre ans après la plantation (fin 1972) fournissent les résultats reproduits au tableau 5 .

On peut remarquer que la croissance en hauteur sur le terrain est conditionnée surtout par l'apport de matière organique en pépinière $(\mathrm{T} 10$ et $\mathrm{T} 1 \mathrm{~F} 2>\mathrm{OF} 2)$, mais que la plus forte hauteur totale est obtenue lorsque les apports organiques et minéraux en pépinière ont été combinés (T1F2 > T10 et OF2).

\subsection{2. $-2^{\mathrm{e}}$ cycle.}

Les mesures effectuées deux ans après la plantation (fin 1972) fournissent les résultats reproduits au tableau 6 .

Bien que les différences d'accroissement entre traitements ne soient pas encore (de peu) significatives, on remarque que les plus fortes croissances sont obtenues après apport de matière organique seule (T20 et T10). Quant à la hauteur totale, il nous sera nécessaire d'attendre encore au moins deux ans pour en tirer tous les enseignements.

\section{IV. - DISCUSSION ET COMMENTAIRES}

\section{1. - Taux de minéralisation annuelle de la matière organique du sol}

Rappelons que tous les commentaires ne sauraient être étendus à des conditions autres que les conditions expérimentales retenues (caractéristiques du sol, en particulier sa texture, nature des amendements).

Les résultats mentionnés précédemment montrent :

- qu'il est encore impossible d'estimer ce taux de minéralisation. Les hétérogénéités de répartition de la matière organique en sont probablement la cause; ainsi il n'y a pas de 
TABLEAU 5

Mesures effectuées 4 ans après la plantation ( $1^{\text {er }}(y c l e$ )

Measures on first cycle 4 years after plantation

\begin{tabular}{|c|c|c|c|c|c|c|c|c|}
\hline & \multirow{2}{*}{ T1F2 } & \multirow{2}{*}{$\mathrm{T} 10$} & \multirow{2}{*}{ OF2 } & \multicolumn{2}{|c|}{$\begin{array}{l}\mathrm{F} \text { théorique } \\
\text { theorical } \mathrm{F}\end{array}$} & \multirow{2}{*}{$\begin{array}{l}\text { F calculé } \\
\text { calculated F }\end{array}$} & \multicolumn{2}{|c|}{$\begin{array}{l}\text { Différences significatives } \\
\text { Significative differences }\end{array}$} \\
\hline & & & & $\begin{array}{l}\text { à } 1 \% \\
\text { at } 1 \%\end{array}$ & $\begin{array}{l}\text { à } 5 \% \\
\text { at } 5 \%\end{array}$ & & $\begin{array}{l}\text { à } 1 \% \\
\text { at } 1 \%\end{array}$ & $\begin{array}{l}\text { à } 5 \% \\
\text { at } 5 \%\end{array}$ \\
\hline $\begin{array}{l}\text { Accroissement depuis la plantation }(\mathrm{cm}) \\
\text { Increasing since plantation (in } \mathrm{cm} \text { ) }\end{array}$ & 127,0 & 125,1 & 113,6 & 10,92 & 5,14 & $9,53+$ & $\mathrm{TIF} 2>\mathrm{OF} 2$ & $\mathrm{~T} 10>\mathrm{OF} 2$ \\
\hline $\begin{array}{l}\text { Hauteur totale fin } 1972(\mathrm{~cm}) \\
\text { Total height at the end of } 1972 \text { (in } \mathrm{cm} \text { ) }\end{array}$ & 192,1 & 179,9 & 178,7 & 10,92 & 5,14 & $10,02+$ & $\mathrm{T} 1 \mathrm{~F} 2>\mathrm{OF} 2$ & $\mathrm{~T} 1 \mathrm{~F} 2>\mathrm{T} 10$ \\
\hline
\end{tabular}


TABLEAU 6

Mesures effectuées 2 ans après la plantation ( $2^{\mathrm{e}}$ cycle)

Measures on second cycle 2 years after plantation

\begin{tabular}{|c|c|c|c|c|c|c|c|c|c|c|}
\hline & T20 & T10 & $\mathrm{T} 1 \mathrm{~F} 1$ & $\mathrm{~T} 2 \mathrm{~F}^{\prime} 1$ & OOF2 & $\mathrm{T} 2 \mathrm{~F} 2$ & OF2 & $\mathrm{T} 1 \mathrm{~F} 2$ & $\begin{array}{l}\mathrm{F} \text { théorique à } 5 \% \\
\text { Theorical } \mathrm{F} \text { at } 5 \%\end{array}$ & $\begin{array}{c}\text { F calculé } \\
\text { Calculated F }\end{array}$ \\
\hline $\begin{array}{l}\text { Accroissement depuis la planta- } \\
\text { tation }(\mathrm{cm}) \\
\text { Increasing since plantation } \\
\text { (in } \mathrm{cm})\end{array}$ & 45,8 & 45,4 & 42,7 & 41,8 & 40,1 & 35,8 & 33,2 & 31,2 & 2,52 & 2,15 \\
\hline $\begin{array}{l}\text { Hauteur totale fin } 1972(\mathrm{~cm}) \\
\text { Total height at the end of } 1972 \\
\quad(\text { in } \mathrm{cm})\end{array}$ & 92,1 & 91,0 & 91,8 & 87,0 & 88,5 & 96,5 & 85,2 & 91,9 & 2,52 & 0,66 \\
\hline
\end{tabular}


différence significative, quant à la teneur en matière organique, entre les traitements ayant reçu la dose T2 et celui n'en ayant pas reçu (OF2). Pourtant, théoriquement en 4 ans, et sans apport nouveau, cette teneur devrait baisser d'environ 0,4 et $0,8 \%$ dans le cas respectivement d'un taux de minéralisation de 2 et $4 \%$ par an;

- qu'il est encore impossible d'évaluer le coefficient isohumique de la terre de bruyère utilisée.

Ce n'est donc que dans plusieurs années que l'on pourra connaître la quantité de terre de bruyère à apporter au début de chaque cycle, permettant de maintenir constant le taux d'humus du sol.

Signalons de même que les taux de saturation et les $\mathrm{pH}$ ne varient guère d'un traitement à l'au tre. Ainsi, la gamme des pH (à l'eau) est comprise entre 3,9 et 4,5 (maximum : OOF2) ce qui convient parfaitement pour des semis de résineux.

\section{2. - Différence, entre les 2 cycles, de comportements des plants après installation sur le terrain}

Alors que, pour les plants issus du $1^{\mathrm{er}}$ cycle, T1F2 avait un comportement après plantation aussi bon que celui de T10, il en va tout autrement pour les plants issus du $2^{\mathrm{e}}$ cycle : les traitements comportant F2, et en particulier T1F2 ont le moins fort accroissement de tous.

Ce sont donc les plants les plus grands au moment de la plantation qui se comportent le moins bien sur le terrain; le système radiculaire de ces plants n'est, par ailleurs, pas mieux développé (il l'est peut-être même moins bien) que celui des plants des autres traitements. Or, les 2 années qui ont suivi la plantation des plants issus du $2^{\text {e }}$ cycle ont été séches, bien plus que les 2 années précédentes. D'où l'hypothèse suivante : la différence entre les 2 cycles serait la conséquence d'un déséquilibre entre parties aériennes et souterraines des plants, déséquilibre à l'origine d'une alimentation en eau insuffisante en année sèche.

On remarque par ailleurs que les concentrations foliaires en phosphore des plants du $2^{\mathrm{e}}$ cycle à la fin des deux années de pépinière sont nettement les moins élevées pour les traitements comportant $\mathrm{F} 2$; il y a peut être déséquilibre $\mathrm{N} / \mathrm{P}$ et, sur le terrain, ces plants n'ont peut-être pas un enracinement suffisant pour prélever assez de phosphore en année sèche.

\section{3. - Utilité de la matière organique dans un sol de pépinière}

En pépinière, OOF2 s'avère nettement inférieur aux autres traitements comportant l'apport F2, et notamment à OF2, pour tous les caractères retenus. Son comportement après plantation ne pourra s'évaluer avant au moins deux ans. Néanmoins, nous pouvons dès à présent affirmer que la présence de matière organique, au départ, dans le sol utilisé et avec les engrais utilisés (engrais azotés très solubles) est très utile : la faible capacité d'échange et la mauvaise stabilité structurale (l'indice Is de OOF2 $=1,06$, celui de OF2 $=0,51$ ) rendent le comportement des plants trop variables suivant les conditions climatiques.

Un apport de matière organique au début de chaque cycle est donc utile, ne serait-ce que pour maintenir la teneur en humus du sol à une valeur raisonnable. Mais il apparaît 
que cet apport joue également un autre rôle; en effet bien que la teneur globale du sol en matière organique ne soit pas en cause (cf. paragraphe 4.1.), un apport de terre de bruyère en pépinière s'est avéré très utile après plantation (accroissement et hauteur totale). Quelle en est la cause? La terre de bruyère, bien que sans doute en grande partie humifiée, contient une certaine proportion de matière organique fraîche, rapidement minéralisable, qui favorise peut-être la vie microbienne et l'enracinement.

\section{4. - Les éléments minéraux : apports par la matière organique et les engrais}

Il n'est pour le moment pas possible de faire un bilan quant à l'utilisation par les plants des éléments libérés par la minéralisation de l'humus; en effet la terre de bruyère ne constitue encore qu'une faible partie de l'humus du sol, alors que les doses $F 1$ et $F^{\prime} 1$ du $2^{\text {e }}$ cycle ont été calculées en fonction des teneurs en éléments nutritifs de la terre de bruyère.

\subsection{1. - Comportement des plants en pépinière.}

Les doses $\mathrm{F} 1$ et $\mathrm{F}^{\prime} 1$ du $2^{\mathrm{e}}$ cycle, compléments respectivement de $\mathrm{T} 1$ et $\mathrm{T} 2$, sont actuellement insuffisantes pour obtenir la croissance la plus importante. Par contre, les doses F1 et $\mathrm{F}^{\prime} 1$ du $1^{\mathrm{er}}$ cycle sont suffisantes. Ainsi, $\mathrm{F}^{\prime} 1$ apporte environ la moitié de l'azote et le tiers du phosphore et du potassium de la dose F2 : il est donc possible et même conseillé dans un sol de pépinière qui contient suffisamment d'humus (par exemple $5 \%$ ) de n'apporter au sol sous forme minérale qu'une fraction des éléments nutritifs qui seront prélevés et exportés; celà évidemment à condition d'effectuer périodiquement des apports de matière organique, destinée à maintenir constant le taux d'humus, et qui, par minéralisation, fournira des éléments nutritifs aux plants.

\subsection{2. - Comportement après plantation et synthèse.}

La dose d'engrais F2 en pépinière ne s'est pas avérée utile pour la croissance de départ sur le terrain; elle est même néfaste en années sèches ( $2^{\mathrm{e}}$ cycle, cf. paragraphe 4.2.). Par contre elle a été utile pour la hauteur totale des plants du $1^{\text {er }}$ cycle après 4 années de plantation (les 2 années ayant suivi la plantation n'ayant pas été sèches). Les doses F1 et $\mathrm{F}^{\prime} 1$ du $1{ }^{\text {er }}$ cycle auraient probablement été suivies du même effet que la dose F2, les plants, au sortir de pépinière, ayant à peu près les mêmes caractéristiques.

Ces résultats sont un peu contradictoires. Ils laissent subsister un certain doute quant à l'intérêt de produire dans tous les cas en pépinière de grands plants par apport de doses assez importantes d'engrais minéraux. Il est cependant possible qu'un équilibre différent entre éléments apportés par fertilisation, favorisant $\mathrm{P}$ par rapport à $\mathrm{N}$, permette de généraliser l'intérêt des grands plants (cf. paragraphe 4.2), mais ce n'est pas certain si les conditions d'alimentation en eau sont critiques après la plantation.

\section{5. - Test de pépinière destiné à juger l'intérêt du traitement}

Cette expérience a montré que la mesure de l'accroissement en hauteur, mesure facile et rapide, constitue un test satisfaisant pour juger de l'ensemble des caractères aériens 
essentiels des plants en pépinière. En effet, les résultats concernant la croissance, la nutrition et les caractères foliaires se rejoignent. Remarquons à ce propos que la taille des feuilles, synthétisant bien l'ensemble des caractères foliaires pour chaque traitement et en accord avec la pousse, traduit finalement une partie des potentialités physiologiques conditionnant le développement des plants.

Nous venons cependant de voir qu'il y a un léger doute quant à l'intérêt d'obtenir de grands plants en pépinière. Il est donc fort possible que la mesure des croissances en hauteur en pépinière ne soit pas un test parfait ou suffisant pour prédire le comportement des sujets après plantation; peut-être devrait-on rechercher un test fondé sur l'appareil racinaire ou l'analyse foliaire, complémentaire de celui de la croissance du système aérien.

\section{V. - CONCLUSIONS}

Rappelons qu'il s'agit d'une expérience à long terme dont nous ne pourrons recueillir tous les renseignements que dans plusieurs années.

Certains résultats pratiques peuvent cependant dès à présent être considérés comme acquis (dans des conditions comparables à celles de l'expérience) :

- la présence d'une certaine quantité de matière organique dans le sol de pépinière est indispensable;

- des apports de matière organique au début de chaque cycle s'avèrent nettement utiles pour la croissance après plantation sur le terrain;

- dans ces conditions, l'apport d'éléments nutritifs sous forme d'engrais minéraux peut être nettement inférieur aux exportations.

Les autres résultats apparaissant dans des conclusions partielles ont été commentés et discutés; ils sont l'objet de travaux en cours.

Reçu pour publication en novembre 1973.

\section{SUMMARY}

INFLUENCE OF ORGANIC MATTER AND MINERAL FERTILIZERS IN NURSERY ON GROWTH AND FOLIAR FEATURES OF DOUGLAS FIR

An experiment including various rates of organic matter $(\mathrm{OO}=$ absence; $\mathrm{O}=$ présence of humus; $\mathrm{T} 1=$ presence of humus $+160 \mathrm{~kg}$ of dry heather soil; $\mathrm{T} 2=$ presence of humus + double of T1) combined with various rates of fertilizers $(\mathrm{O}=$ no fertilizer; $\mathbf{F} 2=$ addition of an amount of fertilizer corresponding to all the elements contained in the plants when they are rooted out; $\mathrm{F} 1$ and $\mathrm{F}^{\prime} 1$ : addition of an amount of fertilizer taking into account the elements of $\mathrm{T} 1$ and $\mathrm{T} 2$ respectively) has been carried out on young douglas-firs in a nursery. (Denomination of the treatments $=$ organic matter + fertilizer; e.g. : OOF2 $=\mathrm{OO}+\mathrm{F} 2$ ).

The purpose of the experiment is to point out the part played by the organic matter not taking into account the fertilizers which it lets loose, to define to what extent those elements allow us to reduce the rate of fertilizers, and to determine the optimal rate of humus and the way to maintain it. results :

In a nursery this experiment, at the end of two cycles of two years each, entails the following

- All treatments that do not include at the same time organic matter and fertilizers (OOF2; T10; T20) do not allow the maximum growth of the plants; 
- The presence of humus with no addition of organic matter appears to be sufficient at least in the nursery and until the end of the second cycle (OF2 $\simeq$ T1F2 and T2F2);

- As regards the addition of fertilizer, the optimum is situated between F2 and F1 or F'1;

- Four years after being planted in a forest their best growth is obtained by the treatments including an addition of heather-soil (T1F2 and T10 > OF2) but as far as their total heights are concerned $\mathrm{T} 1 \mathrm{~F} 2$ is still better than T10 and OF2.

Moreover his experiment shows that the differences in the growing in height of the plants, in a nursery, are in keeping with the variability according to the treatments of the morphological, anatomical and chemical features of the leaves.

\section{ZUSAMMENFASSUNG}

\section{DER EINFLUSS VON HUMUS UND MINERALDÜNGUNG AUF DAS WACHSTUM UND DIE BLATTMERKMALE DER DOUGLASIE IM PFLANZGARTEN}

Der Pflanzgartenversuch mit verschulten Douglasien kombiniert eine organische und eine Mineraldüngung.

Eine bestimmte Menge an Humus im Boden ist eine unumgängliche Voraussetzung für ein maximales Wachstum im Pflanzgarten während der ersten zwei Zyklen von jeweils zwei Jahren. Eine zusätzliche organische Düngung verbessert den Anwuch serfolg einer Aufforstung.

Das Optimum der Mineraldüngung liegt zwischen der Menge an exportierten Nährstoffen und derselben Nährstoffmenge abzüglich der durch den Humus zusätzlich eingebrachten Nährstoffe.

Es zeigte sich desweiteren in diesem Versuch, dass die Unterschiede des Höhenwachstums im Pflanzgarten mit den morphologischen, anatomischen und chemische Unteschieden in den Blättern zwischen den werschiedenen Behandlungen weitgehend übereinstimmen.

\section{RÉFÉRENCES BIBLIOGRAPHIQUES}

Benzian B., 1965. - Experiments on nutrition problems in forest nurseries. 2 vol. Bull. Forestry Comm. London, 37. $251+265$

BenZIAN B., 1966. - Manuring young conifers : experiments in some english nurseries. Fertilizer society Proceedings, 94, 3-37.

Bogar G.D. et Smith F. H., 1965. - Anatomie of seedling root of Pseudotsuga menziesii. Amer. J. Botany, 52, 7, 720-29.

Bonneau M., 1969. - La fertilisation en sylviculture. Revue forestière $F$. no spécial 1969, 429-39.

FLORIN R., 1931. - Untersuchungen zur stammgeschite der Coniferales und Cordaitales. Erster Teil, 1 vol., 58 tab., 111 fig. Kung. Svenska Vet. Akad. Handl., Stockholm. 3 sér., 10, 1, 588.

KRAJINA V. J., 1959. - Ecological requirements of Douglas-fir, western hemlock, Sitka spruce and western red cedar. Natl. Res. Council Progr. Rept. Univ. B. C. on Ecology of forests of the Pacific North West for 1958 .

LÉvy G., 1968. - Importance des propriétés du sol pour l'enracinement de Picea excelsa et de Pinus silvestris. Ann. Sci. forest., 25, 3, 157-88.

Strullu D. G., 1972. - Recherches sur le comportement du Douglas en pépinière (Pseudotsuga menziesii (Mirb.) Franco). (Thèse $3^{\mathrm{e}}$ cycle 1970). Botanica Rhedonica, n ${ }^{\circ} 12,108$.

VAN Den Driessche R., 1963. - Nursery experiments with Douglas-fir. Common. For. Rev., 42, 113, 242-54.

VAN DEN Driessche R., 1967. - A comparaison of responses of Douglas-fir and Sitka spruce to different nitrogen, phosphorus and potassium levels in sand culture. Canad. J. Bot., 46, 531-7. 
ANNEXE I

Caractères foliaires: Moyennes par traitement

\begin{tabular}{|c|c|c|c|c|c|c|c|c|}
\hline & OOF2 & OF2 & T10 & TIF1 & $\mathrm{T} 1 \mathrm{~F} 2$ & $\mathrm{~T} 20$ & $\mathrm{~T} 2 \mathrm{~F}^{\prime} 1$ & $\mathrm{~T} 2 \mathrm{~F} 2$ \\
\hline $\begin{array}{r}\mathbf{X}_{1} \\
\mathbf{X}_{1} \\
(\mathrm{~cm})\end{array}$ & $\begin{array}{l}1,96 \\
2,45\end{array}$ & $\begin{array}{l}2,67 \\
3,45\end{array}$ & $\begin{array}{l}2,73 \\
3,61\end{array}$ & $\begin{array}{l}3,19 \\
3,51\end{array}$ & $\begin{array}{l}3,26 \\
3,77\end{array}$ & $\begin{array}{l}2,83 \\
3,57\end{array}$ & $\begin{array}{l}2,90 \\
3,45\end{array}$ & $\begin{array}{l}2,65 \\
3,52\end{array}$ \\
\hline $\begin{array}{c}\mathrm{X}_{2} \\
\mathrm{X}_{2} \\
(1 / 10 \mathrm{~mm})\end{array}$ & $\begin{array}{l}3,95 \\
6,77\end{array}$ & $\begin{array}{l}4,38 \\
7,09\end{array}$ & $\begin{array}{l}4,32 \\
6,80\end{array}$ & $\begin{array}{l}5,15 \\
6,83\end{array}$ & $\begin{array}{l}4,74 \\
7,06\end{array}$ & $\begin{array}{l}4,43 \\
7,22\end{array}$ & $\begin{array}{l}4,82 \\
7,09\end{array}$ & $\begin{array}{l}4,67 \\
6,77\end{array}$ \\
\hline $\begin{array}{c}\mathrm{X}_{3} \\
\mathbf{X}_{3}\end{array}$ & $\begin{array}{l}1,18 \\
1,52\end{array}$ & $\begin{array}{l}1,27 \\
1,69\end{array}$ & $\begin{array}{l}1,26 \\
1,66\end{array}$ & $\begin{array}{l}1,42 \\
1,62\end{array}$ & $\begin{array}{l}1,32 \\
1,70\end{array}$ & $\begin{array}{l}1,30 \\
1,72\end{array}$ & $\begin{array}{l}1,31 \\
1,63\end{array}$ & $\begin{array}{l}1,26 \\
1,62\end{array}$ \\
\hline $\begin{array}{c}\mathrm{X}_{4} \\
\mathrm{X}_{4} \\
(1 / 10 \mathrm{~mm})\end{array}$ & $\begin{array}{l}0,92 \\
1,18\end{array}$ & $\begin{array}{l}1,02 \\
1,25\end{array}$ & $\begin{array}{l}0,99 \\
1,25\end{array}$ & $\begin{array}{l}1,12 \\
1,25\end{array}$ & $\begin{array}{l}1,05 \\
1,27\end{array}$ & $\begin{array}{l}1,03 \\
1,28\end{array}$ & $\begin{array}{l}1,03 \\
1,23\end{array}$ & $\begin{array}{l}0,99 \\
1,21\end{array}$ \\
\hline $\begin{array}{c}X_{5} \\
X_{5} \\
(1 / 10 \mathrm{~mm})\end{array}$ & $\begin{array}{l}1,97 \\
2,20\end{array}$ & $\begin{array}{l}2,11 \\
2,32\end{array}$ & $\begin{array}{l}1,83 \\
2,45\end{array}$ & $\begin{array}{l}2,15 \\
2,20\end{array}$ & $\begin{array}{l}2,21 \\
2,36\end{array}$ & $\begin{array}{l}1,96 \\
2,42\end{array}$ & $\begin{array}{l}1,92 \\
2,55\end{array}$ & $\begin{array}{l}1,99 \\
2,60\end{array}$ \\
\hline $\begin{array}{c}X_{6} \\
X_{6} \\
(1 / 10 \mathrm{~mm})\end{array}$ & $\begin{array}{l}5,17 \\
8,80\end{array}$ & $\begin{array}{r}5,84 \\
10,03\end{array}$ & $\begin{array}{l}6,18 \\
9,72\end{array}$ & $\begin{array}{l}7,24 \\
9,82\end{array}$ & $\begin{array}{r}6,45 \\
10,17\end{array}$ & $\begin{array}{r}5,99 \\
10,03\end{array}$ & $\begin{array}{l}6,70 \\
9,41\end{array}$ & $\begin{array}{l}6,00 \\
9,15\end{array}$ \\
\hline $\begin{array}{l}\mathrm{x}_{7} \\
\mathrm{X}_{7}\end{array}$ & $\begin{array}{l}113 \\
142\end{array}$ & $\begin{array}{l}108 \\
176\end{array}$ & $\begin{array}{l}107 \\
162\end{array}$ & $\begin{array}{l}120 \\
171\end{array}$ & $\begin{array}{l}107 \\
162\end{array}$ & $\begin{array}{l}108 \\
161\end{array}$ & $\begin{array}{l}112 \\
162\end{array}$ & $\begin{array}{l}104 \\
154\end{array}$ \\
\hline $\begin{array}{l}\mathbf{X}_{8} \\
\mathbf{X}_{8}\end{array}$ & $\begin{array}{l}11,40 \\
15,66\end{array}$ & $\begin{array}{l}12,06 \\
19,66\end{array}$ & $\begin{array}{l}12,00 \\
17,46\end{array}$ & $\begin{array}{l}13,86 \\
19,06\end{array}$ & $\begin{array}{l}12,60 \\
19,20\end{array}$ & $\begin{array}{l}11,46 \\
17,60\end{array}$ & $\begin{array}{l}12,73 \\
17,46\end{array}$ & $\begin{array}{l}11,66 \\
17,40\end{array}$ \\
\hline $\begin{array}{r}\mathbf{X}_{9} \\
\mathbf{X}_{9} \\
(\mathrm{en} \mu)\end{array}$ & $\begin{array}{l}186 \\
295\end{array}$ & $\begin{array}{l}200 \\
320\end{array}$ & $\begin{array}{l}198 \\
304\end{array}$ & $\begin{array}{l}224 \\
299\end{array}$ & $\begin{array}{l}217 \\
317\end{array}$ & $\begin{array}{l}205 \\
288\end{array}$ & $\begin{array}{l}214 \\
304\end{array}$ & $\begin{array}{l}211 \\
331\end{array}$ \\
\hline $\begin{array}{r}\mathbf{X}_{10} \\
\mathbf{X}_{10} \\
(\mathrm{en} \mu)\end{array}$ & $\begin{array}{l}42 \\
81\end{array}$ & $\begin{array}{l}43 \\
88\end{array}$ & $\begin{array}{l}45 \\
87\end{array}$ & $\begin{array}{l}53 \\
82\end{array}$ & $\begin{array}{l}52 \\
93\end{array}$ & $\begin{array}{l}47 \\
96\end{array}$ & $\begin{array}{l}50 \\
97\end{array}$ & $\begin{array}{l}50 \\
91\end{array}$ \\
\hline $\begin{array}{l}\mathrm{X}_{11} \\
\mathrm{X}_{11}\end{array}$ & $\begin{array}{l}14,73 \\
26,86\end{array}$ & $\begin{array}{l}12,60 \\
37,20\end{array}$ & $\begin{array}{r}9,26 \\
29,80\end{array}$ & $\begin{array}{l}13,73 \\
52,53\end{array}$ & $\begin{array}{l}13,53 \\
41,66\end{array}$ & $\begin{array}{l}14,26 \\
36,06\end{array}$ & $\begin{array}{l}12,53 \\
43,40\end{array}$ & $\begin{array}{l}12,33 \\
43,73\end{array}$ \\
\hline $\begin{array}{l}\mathrm{X}_{12} \\
\mathrm{X}_{12}\end{array}$ & $\begin{array}{l}17,53 \\
20,80\end{array}$ & $\begin{array}{l}18,13 \\
24,53\end{array}$ & $\begin{array}{l}16,86 \\
22,00\end{array}$ & $\begin{array}{l}18,26 \\
23,73\end{array}$ & $\begin{array}{l}18,40 \\
23,33\end{array}$ & $\begin{array}{l}18,00 \\
22,40\end{array}$ & $\begin{array}{l}18,26 \\
22,26\end{array}$ & $\begin{array}{l}17,73 \\
22,26\end{array}$ \\
\hline
\end{tabular}

$x$ : feuilles de $1^{\text {re }}$ année

$\mathrm{X}$ : feuilles de $2^{\mathbf{e}}$ année

Caractères :

1 - longueur des feuilles

2 - épaisseur des feuilles

3 - largeur des feuilles

4 - largeur de l'épiderme inférieur

5 - largeur de la nervure

6 - largeur des bandes stomatiques

7 - densité des stomates

8 - nombre de rangées de stomates

9 - hauteur du cylindre central

10 - taille des canaux résinifères

11 - nombre des cellules de l'hypoderme

12 - nombre de cellules de l'endoderme 